\title{
Ein Fall von Osteogenesis imperfecta mit verbreiteten Gefässverkalkungen
}

\author{
von \\ Sven Johansson \\ (Tabula II Fig. 3 und Tabula III)
}

Die oben erwähnte Krankheit ist zwar bei weitem nicht gewöhnlich, ich würde doch nicht einen einzigen Fall als beschreibenswert ansehen, besonders nicht da dieser Fall nicht zu dem Klarlegen der Aetiologie geeignet ist, wenn nicht der fragliche Fall in gewissen Beziehungen sehr eigenartig wäre, ja, so viel ich von der Litteratur gesehen habe, vollständig unik. Krankengeschichte und übrige Befunde sind kurz folgende: N:r 255/1916. P. L. Knabe, 6 Tage alt.

Erblich ist nichts von Interesse. In der Anamnese der Eltern giebt es nichts, dass auf Lues deuten könnte. Der Knabe ist etwas $z u$ früh geboren. Gewicht bei der Geburt c:a $2 \mathrm{Kg}$. Ein paar Tage nach der

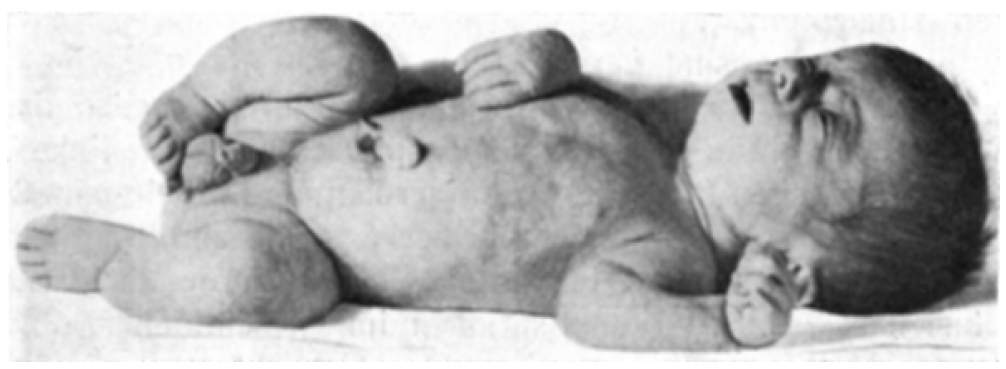

Fig. 1.

Geburt wurde konstatiert, dass der eine Humerus frakturiert war, weshalb das Kind in das Krankenhaus eingenommen wurde.

Status beim Einkommen: Das Kind ist ein venig atrofisch. Gewicht $2280 \mathrm{Gr}$. Das allgemeine Aussehen des Kindes geht übrigens aus dem Bilde 1 hervor. Beide Humeri sind frakturiert. Wasserman nèg. Rönt2 - Acta Radiologica. Vol. I. 1921. 
genphoto einige Tage nach der Ankunft giebt folgendes an die Hand: Das Cranium: Die Basis des Schädels wie der Ober-und Unterkiefer geben deutlichen Knochenschatten. Im übrigen giebt es nur ein sclıwaches Andeuten an Ossifikation in der Form von mosaikenartig angeordneten kleinen Knochenplatten, die am deutlichsten auf dem Gebiete der Ossa parietalia und des Os occipetale hervortreten.

Die oberen Extremitäten: keine Epiphysenkerne. Die Knochen sind arm an Kalk; die Kortikalis ist dünn mit scharfer Kontur; die Spongiosa ohne Struktur; Fraktur der beiden Humeri nahe der proximalen Enden derselben; Fraktur des rechten Radius.

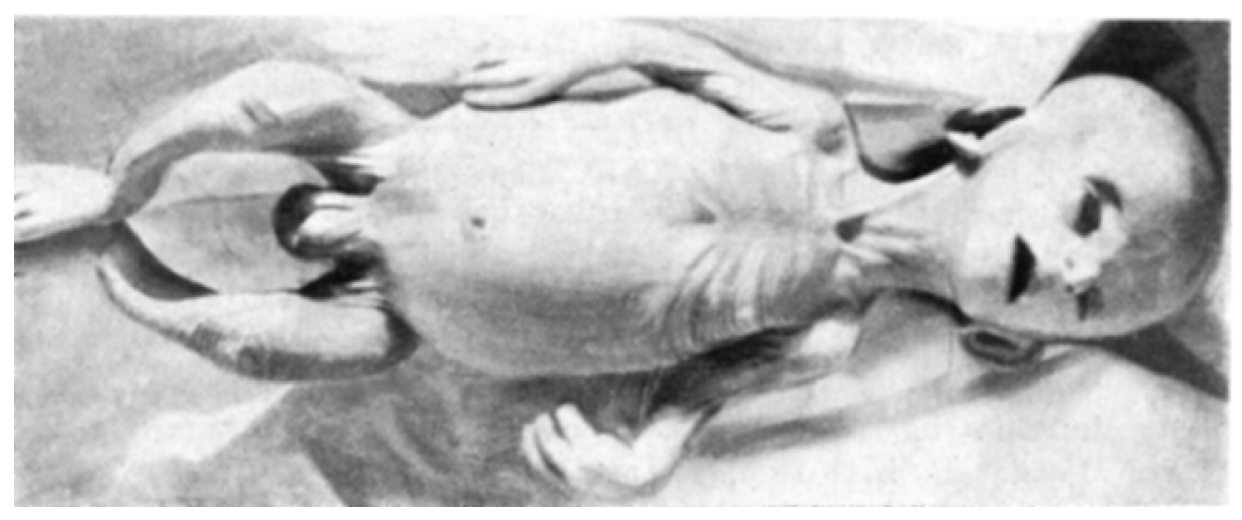

Fig. 2.

Die unteren. Extremitäten: Knochenkerne in der unteren Femur- und der oberen Tibiaepiphyse.

Die Epiphysenlinien sind gerade.

Die beiden oberen Femurenden zeigen Frakturen, ebenso das rechte untere Femurende gerade oberhalb der Epiphysenlinie. Die linke Tibia ist sowohl unten wie oben frakturiert. Ausserdem sind mindestens 2 Rippen gebrochen. Sonst nichts besonders bemerkenswert am Röntgenbilde.

Während des Aufenthaltes im Krankenhause wurde der Zustand des Kindes ohne nachweisbare Komplikationen immer schlimmer, obwohl das Kind von der Mutter ernährt wurde. Das Gewicht sank allmählich von 2280 Gr. zu 1800 Gr. Die Atrophie nahm zu. (Fig. 2). Die ganze Zeit war die Temperatur subnormal, während längerer Zeit c:a $35^{\circ} \mathrm{C}$ und ein paar mal 33.3. ${ }^{\circ} \mathrm{C}$ (!). Mors c:a 3 Monate nach der Ankunft.

Die Sektion zeigte eine Gehirnblutung in dem rechten Temporallobus. Die Thyreoidea und das Thymus sind auffallend klein. Im übrigen nichts besonders bemerkenswertes. Das Aussehen post mortem geht aus dem Bilde 2 hervor. 
Röntgenphoto post mortem zeigt folgendes:

Das Cranium: Keine augenfällige Veränderung im Vergleich mit dem vorigen Bilde.

Die oberen Extremitäten: Die eine Humerusfraktur ist geheilt, bei der anderen hat die Heilung angefangen, doch mit sehr geringer Callusbildung. Die Radiusfraktur ist geheilt. Die Corticalis ist dünn und scharf gezeichnet; anfangende Spongiosazeichung. Auf der rechten Seite sieht man einen kleinen Knochenkern in der oberen Humerusepiphyse. Am rechten Arm tritt sowohl am Ober- wie am Unterarm deutlich ein leicht geschlungenes Gefäss hervor (Arteria brachialis und radialis.) Fig. 3. Tab. II.

Die unteren Extremitäten: Auch hier sind die Frakturen geheilt. Das linke obere Femurende ist stark aufgetrieben. Distinkte, gerade Epiphysenlinien. An beiden Beinen (besonders am linken) tritt deutlich ein leicht gewundenes grosses Gefäss hervor, sowohl am Oberschenkel wie am Unterbein (Arteria femoralis und tibialis). Fig. 1. Tab. III. Die Unterbeine sind stark gekrümmt. Ausserdem nichts bemerkenswertes, wenn nicht ein paar geheilte Rippenfrakturen.

Mikroskopische Untersuchung der Weichteile der Fossa poplitea: Auf dem Schnitt Muskulatur, Nerven, Artären und Venen. Hyperplasie der Intima der sämtlichen grossen und mittelgrossen Artären. In den mittelgrossen Artären ist die Hyperplasie von konzentrischem Typus und die Media unverändert. In den grössten Artären ist die Intimaverdickung zu der einen Seite der Gefässwand lokalisiert. Die Media ist hier in grosser Ausdehnung zerstört, das Gewebe von grossen Kalkschollen ersetzt. Die Elastica intimae, ebenso kalkincrustiert, scheint die degenerativen Veränderungen gegen das Gefässlumen abzugrenzen. Die Mediazerstörung ist am meisten auf dem Gebiete ausgesprochen, welches der stärksten Intimaverdickung entspricht. Die Wände der Venen zeigen keine Veränderungen. Das Gevebe der Gefässwände im übrigen befindet sich in einem ziemlich embryonalen Stadium mit geringer Differentierung. (Dr. G. OdELBERG-Jonson.)

Epikrise: Es ist kaum möglich zu bezweifeln, dass der hier vorliegende Fall zu der heutzutage sowohl klinisch wie pathologisch-anatomisch gut abgegrenzten Krankheitsgruppe gezählt werden muss, welche Osteogenesis imperfecta genannt wird. Das allgemeine Aussehen, der klinische Verlauf, die Röntgenbilder, alles spricht dafür. Denjenigen, welcher vielleicht nicht diese Arbeit kennt, möchte ich in diesem Zusammenhang auf die ausgezeichnete Monographie von Frangenheim hinweisen, über -Die Krankheiten des Knochensystems im Kindesalter» (Neue Deutsche Chirurgie, Bd 10), und kann, wenn ich hierauf hinweise, von weiterem Räsonnieren betreffs der Differentialdiagnose etz. abstehen. Was in 
diesem. Falle besonders anmerkungswert ist und was mich auch veranlasst hat denselben zu publizieren, ist das Vorkommen von Kalk in den Gefässen und dieses in so grosser Menge, dass die Gefässe deutlich auf dem Röntgenbilde hervortreten.

Wie interessant es auch wäre die Frage von der Pathogenese zur Diskussion aufzunehmen und besonders die Ursache zu der Kalkeinlagerung in den Gefässen, zu dem Mangel an Kalk im Skelett in Parietät gestellt, muss ich doch davon abstehen; hierzu wären viel mehr eingehende histologische Untersuchungen von Nöten, als diejenige zu dessen Ausführen ich Gelegenheit gehabt habe. Ich möchte es doch nicht unterlassen diesen Fall denjenigen mitzuteilen die mit der Lösung solcher Probleme beschäftigt sind. Die geringe Entwickelung der Thyreoidea und des Thymus muss notiert werden. Frühere Forscher haben keine Veränderungen in diesen Organen nachweisen können, die mit der Krankheit in pathogenetischem Zusammenhang gesetzt werden konnten. Die Gehirnblutung, die wohl die nächste Todesursache war, muss nicht auf das Konto der Gefässveränderungen geschrieben werden, wenn auch solche im Gehirn vorhanden waren, welches leider nicht untersucht worden ist; man hat nähmlich bei Osteogenesis imperfecta ziemlich oft Gehirnblutungen gesehen, welche ganz genügend von den unvollständigen Ossifikation der Kranialknochen erklärt werden sowie durch das damit in Verbindung stehende Unvermögen gegen Traumata zu schützen.

Das Verhältnis, dass die Gefässveränderungen nicht auf dem ersten Röntgenbilde nachweisbar waren, darf nicht so gedeutet werden, als seien dieselben damals nicht vorhanden, sondern beruht gewiss auf die bei dieser Gelegenheit weniger scharf gezeichneten Bilder.

\section{Zusammenfassung}

Verf. beschreibt einen. Fall von Osteogenesis imperfecta mit multiplen Frakturen, welcher von besonderem Interesse ist, weil die Arterien sowohl der oberen wie der unteren Extremitäten auf den R. bildern deutlich hervortretende arterioscleriotische Veränderungen zeigen. Histologische Untersuchungen zeigten Degeneration und Kalkinkrustationen der Intima und Media der Arterien. Gl. Thyreoidea und Thymus auffallend klein. Kein ähnlicher Fall ist, so viel der Verf. weiss, beschrieben worden. 